\title{
The Effect of Initial Oxygen Exposure on Diaphragm Activity in Preterm Infants at Birth
}

\begin{abstract}
Ruud W. van Leuteren ${ }^{1,2 *+}$, Anouk W. J. Scholten ${ }^{1,2+}$, Janneke Dekker ${ }^{3}$, Tessa Martherus ${ }^{3}$, Frans H. de Jongh ${ }^{1,4}$, Anton H. van Kaam ${ }^{1,5}$, Arjan B. te Pas ${ }^{3}$ and Jeroen Hutten ${ }^{1,5}$

${ }^{1}$ Department of Neonatology, Emma Children's Hospital, Amsterdam UMC, University of Amsterdam, Amsterdam, Netherlands, ${ }^{2}$ Amsterdam Reproduction and Development Research Institute, Amsterdam, Netherlands, ${ }^{3}$ Department of Neonatology, Leiden University Medical Centre, Leiden, Netherlands, ${ }^{4}$ Faculty of Science and Technology, University of Twente, Enschede, Netherlands, ${ }^{5}$ Department of Neonatology, Emma Children's Hospital, Amsterdam UMC, Vrije Universiteit, Amsterdam, Netherlands
\end{abstract}

OPEN ACCESS

Edited by:

Gianluca Lista,

Ospedale dei Bambini Vittore

Buzzi, Italy

Reviewed by:

Carlo Dani,

University of Florence, Italy

Mark Prutkin,

Irkutsk Regional Pediatric

Hospital, Russia

Camilla Gizzi,

Ospedale San Carlo, Italy

*Correspondence:

Ruud W. van Leuteren r.w.vanleuteren@amsterdamumc.nl

†These authors have contributed equally to this work and share first authorship

Specialty section:

This article was submitted to Neonatology,

a section of the journa Frontiers in Pediatrics

Received: 11 December 2020 Accepted: 15 January 2021

Published: 09 February 2021

Citation: van Leuteren RW, Scholten AWJ, Dekker J, Martherus T, de Jongh FH, van Kaam AH, te Pas AB and Hutten J (2021) The Effect of Initial Oxygen Exposure on Diaphragm Activity in Preterm Infants at Birth. Front. Pediatr. 9:640491. doi: 10.3389/fped.2021.640491
Background: The initial $\mathrm{FiO}_{2}$ that should be used for the stabilization of preterm infants in the delivery room (DR) is still a matter of debate as both hypoxia and hyperoxia should be prevented. A recent randomized controlled trial showed that preterm infants [gestational age $(\mathrm{GA})<30$ weeks] stabilized with an initial high $\mathrm{FiO}_{2}$ (1.0) had a significantly higher breathing effort than infants stabilized with a low $\mathrm{FiO}_{2}$ (0.3). As the diaphragm is the main respiratory muscle in these infants, we aimed to describe the effects of the initial $\mathrm{FiO}_{2}$ on diaphragm activity.

Methods: In a subgroup of infants from the original bi-center randomized controlled trial diaphragm activity was measured with transcutaneous electromyography of the diaphragm (dEMG), using three skin electrodes that were placed directly after birth. Diaphragm activity was compared in the first $5 \mathrm{~min}$ after birth. From the dEMG respiratory waveform several outcome measures were determined for comparison of the groups: average peak- and tonic inspiratory activity ( $d E M G_{\text {peak }}$ and $d E M G_{t o n}$, respectively), inspiratory amplitude ( $\left.d E M G_{a m p}\right)$, area under the curve ( $\left.d E M G_{A \cup C}\right)$ and the respiratory rate $(\mathrm{RR})$.

Results: Thirty-one infants were included in this subgroup, of which 29 could be analyzed $[n=15$ (median GA 28.4 weeks) and $n=14$ (median GA 27.9 weeks) for the 100 and $30 \%$ oxygen group, respectively]. Tonic diaphragm activity was significantly higher in the high $\mathrm{FiO}_{2}$-group $(4.3 \pm 2.1 \mu \mathrm{V}$ vs. $2.9 \pm 1.1 \mu \mathrm{V} ; p=0.047)$. The other dEMG-parameters (dEMG peak, dEMGamp, $d E M G_{A \cup C}$ ) showed consistently higher values in the high $\mathrm{FiO}_{2}$ group, but did not reach statistical significance. Average RR showed similar values in both groups (34 \pm 9 vs. $32 \pm 10$ breaths/min for the high and low oxygen group, respectively).

Conclusion: Preterm infants stabilized with an initial high $\mathrm{FiO}_{2}$ showed significantly more tonic diaphragm activity and an overall trend toward a higher level of diaphragm activity than those stabilized with an initial low $\mathrm{FiO}_{2}$. These results confirm that a high initial $\mathrm{FiO}_{2}$ after birth stimulates breathing effort, which can be objectified with dEMG.

Keywords: diaphragm activity, breathing effort, oxygen, preterm infant, delivery room 


\section{INTRODUCTION}

At birth, lung liquid clearance and aeration are required to ensure adequate gas exchange after the cord is clamped. Spontaneous breathing is one of the key factors in this process (1). In contrast to term infants, up to $70 \%$ of preterm infants experience difficulty in making this pulmonary transition, with absent or insufficient respiratory drive as an important cause $(2,3)$. For this reason, preterm infants often require continuous positive airway pressure (CPAP) or intermittent positive pressure ventilation (IPPV) in combination with supplemental oxygen in the delivery room (DR) to successfully make the transition from intra- to extrauterine life.

In recent years, various studies have investigated individual interventions to stimulate spontaneous breathing in preterm infants at birth. It has been shown that optimization of the CPAP pressure-level, use of repetitive tactile stimulation, or administering caffein shortly after birth stimulates spontaneous breathing, thereby contributing to a successful transition (4-6). While oxygenation is a major determinant of respiratory drive, the optimal initial fraction of oxygen $\left(\mathrm{FiO}_{2}\right)$ that should be used in the DR is still a matter of debate. Restricted use of oxygen has been advocated in recent guidelines, in an attempt to reduce the risk of injurious hyperoxemia (7). However, using a lower initial $\mathrm{FiO}_{2}$ at birth may also lead to hypoxemia which can inhibit breathing effort and thus pulmonary transition (8). This was recently confirmed by a randomized trial (the IMPROvE trial) assessing the effect of an initial high vs. a low $\mathrm{FiO}_{2}$ on breathing effort in preterm infants (9). The results showed that infants in the high $\mathrm{FiO}_{2}$ (1.0) group had larger tidal volumes, resulting in a significantly higher average minute volume (MV) during the first 5 min after birth, compared to a low $\mathrm{FiO}_{2}(0.3)$. The differences between the two $\mathrm{FiO}_{2}$ groups confirm that oxygen might be an important mediator of breathing effort after birth.

It is known that respiratory control consists of the respiratory rhythm regulator in the brain stem, the sensory input of central and peripheral oxygen chemoreceptors providing feedback to central regulation, and the muscular effector. Extensive evidence exists of the influence of oxygen and neurotransmitters (e.g., adenosine) on the signaling pathways between sensory input of the chemoreceptors and the respiratory rhythm regulator in the brainstem $(8,10)$. On the other hand, the effects of initial oxygen exposure on the muscular effector of the system, the diaphragm, are less clear. As the main effector of the system, the improved breathing effort when administering higher concentrations of oxygen is likely to be mediated through an increased diaphragmatic activity. To investigate this mechanism, we measured the electrical activity of the diaphragm via transcutaneous electromyography (dEMG) in a subgroup of the infants included in the IMPROvE trial. A recent study showed that transcutaneous dEMG monitoring is feasible in preterm infants stabilized in the DR (11).

The aim of this study was to determine the effects of an initial high vs. low oxygen level on the activity of the diaphragm in preterm infants and we hypothesized that a higher oxygen level would increase the electrical activity compared to a lower oxygen concentration.

\section{METHODS}

This study was part of the IMPROvE trial, a randomized controlled trial conducted in the Leiden University Medical Center (LUMC) and Amsterdam University Medical Center (Amsterdam UMC), both located in the Netherlands (9).

\section{Study Population and Data Acquisition}

Preterm infants born after a gestational age between $24^{0 / 7}$ and $29^{6 / 7}$ weeks were included in the IMPROvE trial. Exclusion criteria were congenital abnormalities or conditions that could directly affect breathing effort. All infants were randomly allocated to an initial high (1.0) or low (0.3) $\mathrm{FiO}_{2}$. During the stabilization in the DR (first $15 \mathrm{~min}$ after birth), the oxygen level was titrated according to the Dawson oxygen saturation ranges (12). Respiratory support was provided with a facemask connected to a T-piece resuscitator, following the protocol of the individual departments [based on international guidelines and the Dawson saturation curves $(9,13)]$. A respiratory function monitor (RFM) (NewLifeBox, Advanced Life Diagnostics, Weener, Germany) was used to record respiratory data and vital signs in real time within a Polybench software application (Applied Biosignals, Weener Germany).

In 31 of the 50 included infants, diaphragm activity was measured with dEMG. Three skin electrodes (H59P, Covidien, Ireland) were placed on the infant's chest, two bilateral in the midclavicular line at the costal margin and a reference electrode on the sternum. The electrodes were connected to the Dipha-16 signal amplifier (Demcon, Macawi Medical Systems, Enschede, The Netherlands) which measured the raw dEMG-signal at $500 \mathrm{~Hz}$ and sent it wirelessly to the RFM. Electrodes were placed as soon as possible after birth and measured diaphragm activity for the first $15 \mathrm{~min}$ after birth or until transfer to the ward. A camera placed at the far end of the bed recorded the procedures, in order to explain signal artifacts afterwards.

\section{Data Analysis}

The amplified dEMG signal was post-processed to remove background noise by high-pass and low-pass filtering and cardiac interference was removed with the gating technique (14). The moving average of the resulting signal was calculated and the acquired respiratory waveform was used to describe diaphragmatic activity in the two groups. The entire recording was analyzed, as it offered the opportunity to assess diaphragm activity over time (11). Offline analysis was done in MATLAB (version 2018a, Mathworks, Natick, USA) in a custom-made graphical user interface to facilitate visual inspection of the data.

First, review of the video footage, visual inspection of the signals and a threshold value to identify signal spikes were used to remove major signal artifacts due to e.g., disconnection of the electrode or clinical handling. Subsequently, the cleaned signal was used to derive the following parameters breathby-breath from the dEMG respiratory waveform: peak (endinspiratory, $\mathrm{dEMG}_{\text {peak }}$ ) and tonic (end-expiratory, $\mathrm{dEMG}_{\text {ton }}$ ) activity, inspiratory amplitude $\left(\mathrm{dEMG}_{\mathrm{amp}}\right)$ defined as $\mathrm{dEMG}_{\text {peak }}$ minus previous $\mathrm{dEMG}_{\text {ton }}$, inspiratory area under the curve $\left(\mathrm{dEMG}_{\mathrm{AUC}}\right)$, inspiratory time $\left(\mathrm{T}_{\mathrm{i}}\right)$, expiratory time $\left(\mathrm{T}_{\mathrm{e}}\right)$ and 
respiratory rate (RR). Each parameter was averaged per minute, for each infant. If data of a specific minute was not available, due to artifact removal, this minute was discarded.

\section{Statistical Analysis}

All parametric data were presented as mean \pm standard deviation and non-parametric data as median (interquartile range). In agreement with the original IMPROvE trial differences in average dEMG-parameters in the first 5 min after birth between the study groups were tested with a Student's $t$-test or with Mann-Whitney $U$-test, according to the distribution of the data. The choice of this time frame was also supported by the finding in the original trial that after $5 \mathrm{~min}$ of $\mathrm{FiO}_{2}$ titration the contrast in averaged received $\mathrm{FiO}_{2}$ between the groups was reduced (9). In all cases a $p<0.05$ was considered significant. SPSS (version 26, IBM, Chicago, USA) was used for statistical analysis.

\section{RESULTS}

In 31 infants dEMG was measured, of which measurements of two infants were excluded from the analysis due to an error in data storage. This resulted in 29 infants, who were equally distributed between the high $(n=15)$ and low $(n=14)$ oxygen group (Table 1). There were no significant differences between the two groups with respect to gestational age, weight, gender, mode of delivery, use of antenatal steroids, maternal medication or pregnancy complications. IPPV was used more often in the first $5 \mathrm{~min}$ in the $30 \%$ oxygen group, but this difference did not reach statistical significance. However, infants in the $100 \%$ oxygen group showed a significantly higher Apgar score at 1 and 5 min.

\section{Oxygen and Diaphragm Activity}

As shown in Figure 1 peak and tonic activity were consistently higher in the $100 \%$ group than in the $30 \%$ oxygen group. The average value of $\mathrm{dEMG}_{\text {ton }}$ in the first $5 \mathrm{~min}$ after birth was significantly higher in the $100 \%$ group compared to the $30 \%$ group $(4.3 \pm 2.1 \mu \mathrm{V}$ vs. $2.9 \pm 1.0 \mu \mathrm{V} ; p=0.047)$. The $\mathrm{dEMG}_{\text {peak }}$ value was also higher in the $100 \%$ group compared to the $30 \%$ group but this difference did not reach statistical significance $(10.2 \pm 5.1 \mu \mathrm{V}$ vs. $7.2 \pm 2.7 \mu \mathrm{V}$ for the 100 and $30 \%$ oxygen group, respectively, $p=0.08)$. Average $\mathrm{dEMG}_{\mathrm{amp}}(6.5 \pm 4.1 \mu \mathrm{V}$ vs. $4.2 \pm 2.5 \mu \mathrm{V} ; p=0.11)$ and $\mathrm{dEMG}_{\mathrm{AUC}}(1.8 \pm 1.0 \mu \mathrm{V} \cdot s$ vs. $1.3 \pm 0.7 \mu \mathrm{V} \cdot s ; p=0.19)$ did not show statistically significant differences in the first $5 \mathrm{~min}$ after birth, between the high and the low oxygen group.

With respect to the dEMG derived breathing pattern, the two groups did not show differences in inspiratory and expiratory time. Overall respiratory rate was also similar in both groups (average mean RR in the first 5 min $34 \pm 9$ vs. $32 \pm 10$ breaths/min for the 100 and $30 \%$ oxygen group, respectively, $p=0.47)$.

\section{DISCUSSION}

In this study we demonstrated that a high level of oxygen administered to preterm infants directly after birth resulted in a
TABLE 1 | Subject characteristics of the dEMG-subgroup.

\begin{tabular}{lcc}
\hline & $\mathbf{1 0 0} \% \mathbf{O}_{\mathbf{2}} \boldsymbol{n}=\mathbf{1 5}$ & $\mathbf{3 0} \% \mathbf{O}_{\mathbf{2}} \boldsymbol{n}=\mathbf{1 4}$ \\
\hline Gestational age (weeks) & $28.4(26.1-29.0)$ & $27.9(26.3-28.6)$ \\
Birth weight (g) & $1,060 \pm 314$ & $991 \pm 253$ \\
Male gender, $n$ (\%) & $7(53.3)$ & $6(42.9)$ \\
Cesarean section, $n$ (\%) & $11(73.3)$ & $7(50.0)$ \\
Full course antenatal & $7(46.7)$ & $8(57.1)$ \\
corticosteroids, $n$ (\%) & & \\
Maternal medication & $1(6.7)$ & $0(0)$ \\
influencing infant respiration, & & \\
$n$ (\%) & $6(40.0)$ & $5(35.7)$ \\
Complications during & & \\
pregnancy, $n$ (\%) & $1(6.7)$ & $1(7.1)$ \\
PPROM & $3(20.0)$ & $1(7.1)$ \\
PIH & $0(0)$ & $1(7.1)$ \\
Intra-uterine infection & $0(0)$ & $2(14.3)$ \\
IUGR & $2(13.3)$ & $5(0)$ \\
Multiple & $6(6-8)$ & $8(6-6)^{\star}$ \\
Apgar 1 min & $8(8-9)$ & $26.8(3.6-49.8)$ \\
Apgar 5 min & $10.9(0-30.4)$ & \\
IPPV (\% of first 5 min) & &
\end{tabular}

All continuous values are expressed as mean \pm standard deviation or median (interquartile range). Categorical variables expressed as $n$ (\%). PPROM, preterm pre-labor rupture of membranes; $P I H$, pregnancy-induced hypertension; IUGR, intra-uterine growth restriction; IPPV, intermittent positive pressure ventilation. ${ }^{*} p<0.05$.

consistently higher level of diaphragm activity compared to a low $\mathrm{FiO}_{2}$. No differences were found in inspiration time, expiration time, and respiratory rate.

We hypothesize that the higher diaphragmatic activity in the $100 \%$ group may, in part, explain the previously reported higher tidal volumes when administering $100 \%$ of oxygen compared to $30 \%$ of oxygen (9). First, both the higher peak and tonic diaphragmatic activity in the 100\% oxygen group may assist in lung aeration after birth. A higher peak diaphragmatic activity could facilitate air entry in the fluid-filled lung during the first minutes of life. On top of that, an increased tonic diaphragmatic activity has been associated with an improved functional residual capacity (FRC) and thus retainment of inhaled air during pulmonary transition $(15,16)$. As shown by pre-clinical studies, more effective lung aeration can result in higher tidal volumes (17). Second, the higher diaphragmatic activity itself affects tidal volume. A recent study exploring the effect of caffeine on diaphragmatic activity and tidal volume showed a positive association between these two parameters (18).

Other non-diaphragm related factors may also contribute to a higher tidal volume when exposing infants to a higher $\mathrm{FiO}_{2}$. It has been shown that oxygen supports the development of a more stable breathing pattern and improves coordination of opening and closure of the glottis (19). These factors result in more efficient non-invasive respiratory support, which can impact both tidal volume and FRC.

The effect of oxygen on diaphragm activity was fast as the $100 \%$ oxygen group already had a higher level of diaphragm activity compared to the $30 \%$ group, in the first minutes of 


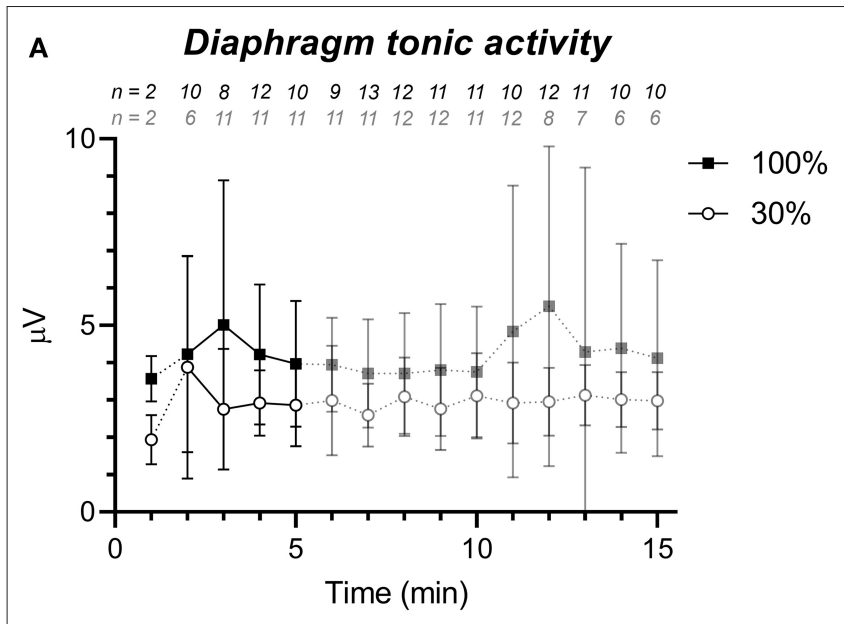

B

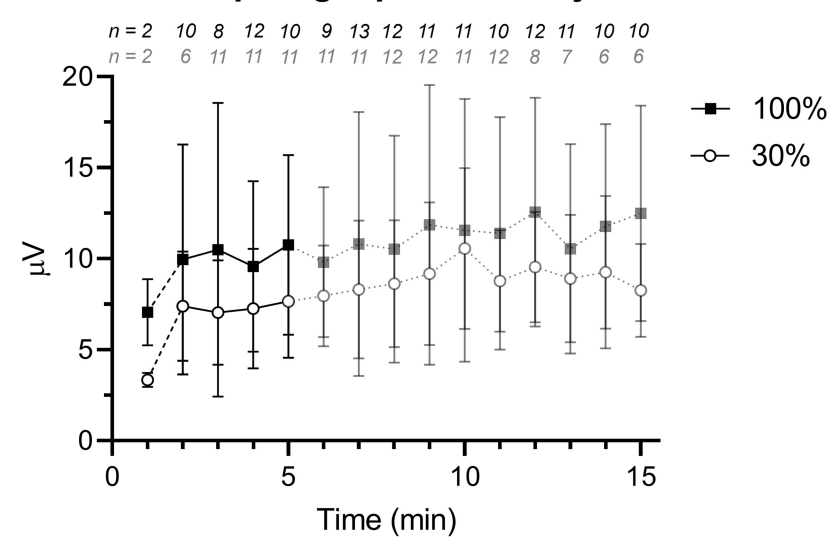

FIGURE 1 | dEMG signal strength parameters (mean \pm standard deviation) for the $30 \%$ (white circles) and 100\% group (black squares) showing a higher level of diaphragm activity in the $100 \%$ group. (A) tonic diaphragm activity and (B) peak diaphragm activity. The first line piece is dashed, due to the low sample size directly after birth. Data after $5 \mathrm{~min}$ is semi-transparent as this data was not taken into account in the statistical analysis. The numbers above the graph represent the number of infants of which dEMG data was available (after artifact removal) for that particular minute and group.

life. Furthermore, mean peak and tonic diaphragmatic activity remained higher on average in the $100 \%$ oxygen group at every time point up to 15 min after birth, even though $\mathrm{dEMG}_{\text {peak }}$ did not reach statistical significance.

The diaphragm activity in the $100 \%$ group remained higher until 15 min after birth, while the difference in the administered $\mathrm{FiO}_{2}$ and $\mathrm{SpO}_{2}$ between the groups decreased over time (9). This might indicate that the effect of the initial oxygen exposure persists after weaning of the $\mathrm{FiO}_{2}$ in the $100 \%$ group. This effect could be a direct result of the $\mathrm{FiO}_{2}$, or indirect (through improved overall oxygenation) as the $100 \%$ group became normoxic earlier in time (9). The administered $\mathrm{FiO}_{2}$ could be sensed by oxygen sensitive neuroendocrine cells present in the pulmonary epithelium, but current evidence on the role of these receptors during the transition from intra- to extra-uterine life is limited (20). It is also known that hypoxia inhibits both the neural output from the brainstem [through e.g., ATP and adenosine pathways (21)] and influences muscle performance itself (22). However, as $\mathrm{FiO}_{2}$ directly affects $\mathrm{SpO}_{2}$ and these parameters go hand in hand with each other it is impossible to determine whether the effect of the initial $\mathrm{FiO}_{2}$ on diaphragm activity is direct or indirect.

In general, the variability in diaphragm activity between infants was high. This finding might explain the lack of significance in $\mathrm{dEMG}_{\mathrm{amp}}$ and $\mathrm{dEMG}_{\mathrm{AUC}}$. There be may several reasons to explain this finding. First, the signal strength may be influenced by the varying electrode-diaphragm distance and skin-electrode interface between infants. The latter is also affected by differences in the wetness of skin between infants, which influences electrode adhesiveness and thereby signal strength (11). Second, similar to the in-utero situation, there is a physiological variation in breathing pattern in preterm infants shortly after birth (23). All these factors contribute to the variability in diaphragm activity between infants and thus make it difficult to obtain reference values for diaphragm activity. Larger studies are needed to determine these normative values. Meanwhile, dEMG can be used to monitor the trend in diaphragm activity over time and to determine the effect of changing the level of respiratory support in the DR.

Studies assessing diaphragmatic activity in the DR are limited. A recent study on the feasibility of dEMG to monitor heart rate and respiratory rate in the delivery room also assessed diaphragmatic activity (11). This study showed a trend to decreasing peak and tonic activity over time. The relatively stable diaphragm activity in the current study does not seem entirely consistent with this feasibility study. However, there were important differences between these studies which may explain this inconsistency. First, infants in the current study were less mature than those included in the feasibility study (mean gestational age 28 vs. 32 weeks). Less mature infants have a more compliant chest wall and therefore need to maintain a high level of muscle activity to open the lungs and keep them open $(11,24,25)$. Second, the trend in diaphragmatic activity in the feasibility study was measured over a longer time $(30 \mathrm{~min})$ and larger intervals $(5 \mathrm{~min})$, which hampers direct comparison.

\section{Study Limitations}

This study has several limitations that need to be addressed. First, there was an inevitable delay in placing the electrodes after birth, which was not part of the normal neonatal resuscitation program. As a result, diaphragmatic activity during the first minute of transition was missed in most of the included infants. However, as this was the case in both oxygen groups, it is unlikely to have biased the results. In addition, it should be mentioned that dEMG was only recorded in case research personnel and equipment were available, which could have introduced a selection bias. Third, although statistically not significant, the $30 \%$-group received more IPPV than the 100\% group, which may have affected the diaphragmatic activity (11). Finally, the unanticipated large variability in diaphragm activity and the relatively small sample size may have compromised the power of our study. Future studies should take this into account. 


\section{Clinical Implication}

The results of this study emphasize the importance of the diaphragm in pulmonary transition. Stimulating diaphragmatic activity with higher concentrations of inspired oxygen seems to facilitate lung aeration and improve tidal ventilation. The concomitant improvement in oxygenation might prove important as hypoxemia $<80 \%$ at $5 \mathrm{~min}$ after birth is associated with a higher risk for mortality and intraventricular hemorrhage (26). Our study results show that diaphragm activity is influenced by the initial level of oxygen exposure. Whether a similar effect on diaphragm activity could be found with an intermediate level of oxygen (e.g., $\mathrm{FiO}_{2}$ 0.5-0.6) might be interesting to investigate, as it could reduce potential oxidative effects. Therefore, evaluation of diaphragm activity could contribute to the discussion on which initial level of oxygen to use in the first minutes of life.

\section{CONCLUSION}

Electrical activity of the diaphragm, measured with transcutaneous electromyography, showed significantly higher tonic activity and a trend to higher peak activity in preterm infants resuscitated with $100 \%$ compared to $30 \%$ oxygen at birth. This finding suggests that the observed improvement in tidal breathing when using $100 \%$ instead of $30 \%$ oxygen is, at least partly, mediated by an oxygen dependent increase in diaphragmatic activity.

\section{REFERENCES}

1. Siew ML, Wallace MJ, Kitchen MJ, Lewis RA, Fouras A, Te Pas AB, et al. Inspiration regulates the rate and temporal pattern of lung liquid clearance and lung aeration at birth. J Appl Physiol. (2009) 106:1888-95. doi: 10.1152/japplphysiol.91526.2008

2. Hooper SB, Te Pas AB, Kitchen MJ. Respiratory transition in the newborn: a three-phase process. Arch Dis Child Fetal Neonatal Ed. (2015) 101:1-7. doi: 10.1136/archdischild-2013-305704

3. Lista G, Maturana A, Moya FR. Achieving and maintaining lung volume in the preterm infant: from the first breath to the NICU. Eur J Pediatr. (2017) 176:1287-93. doi: 10.1007/s00431-017-2984-y

4. Martherus T, Oberthuer A, Dekker J, Kirchgaessner C, van Geloven $\mathrm{N}$, Hooper SB, et al. Comparison of two respiratory support strategies for stabilization of very preterm infants at birth: a matchedpairs analysis. Front Pediatr. (2019) 7:1-10. doi: 10.3389/fped.2019. 00003

5. Dekker J, Hooper SB, Van Vonderen JJ, Witlox RSGM, Lopriore E, Te Pas AB. Caffeine to improve breathing effort of preterm infants at birth: a randomized controlled trial. Pediatr Res. (2017) 82:290-6. doi: 10.1038/pr.2017.45

6. Dekker J, Hooper SB, Martherus T, Cramer SJE, van Geloven N, te Pas AB. Repetitive versus standard tactile stimulation of preterm infants at birth-A randomized controlled trial. Resuscitation. (2018) 127:37-43. doi: 10.1016/j.resuscitation.2018.03.030

7. Soar J, MacOnochie I, Wyckoff MH, Olasveengen TM, Singletary EM, Greif R, et al. 2019 International consensus on cardiopulmonary resuscitation and emergency cardiovascular care science with treatment recommendations: summary from the basic life support; advanced life support; pediatric life support; neonatal life support; education, implementation, and teams; and first aid task forces. Circulation. (2019) 140:E826-80. doi: 10.1161/CIR.0000000000000734

\section{DATA AVAILABILITY STATEMENT}

The datasets generated for this study are available on request to the corresponding author.

\section{ETHICS STATEMENT}

The studies involving human participants were reviewed and approved by Commissie Medische Ethiek, cme registration: P17.209, cme@lumc.nl. Written informed consent to participate in this study was provided by the participants' legal guardian/next of kin. Written informed consent was obtained from the individual(s), and minor(s)' legal guardian/next of kin, for the publication of any potentially identifiable images or data included in this article.

\section{AUTHOR CONTRIBUTIONS}

RL, FJ, AK, JD, AP, and JH conceptualized the study. RL and AS analyzed the data and wrote the first version of the manuscript. RL submitted the paper. All authors contributed to the interpretation of the study results and critically reviewed and contributed to the final draft of the manuscript.

\section{FUNDING}

AP was recipient of an NWO innovational research incentives scheme (VIDI 91716428).

8. Bairam A, Niane LM, Joseph V. Role of ATP and adenosine on carotid body function during development. Respir Physiol Neurobiol. (2013) 185:57-66. doi: 10.1016/j.resp.2012.06.016

9. Dekker J, Martherus T, Lopriore E, Giera M, McGillick EV, Hutten J, et al. The Effect of initial high vs. low $\mathrm{FiO}_{2}$ on breathing effort in preterm infants at birth: a randomized controlled trial. Front Pediatr. (2019) 7:1-11. doi: 10.3389/fped.2019.00504

10. Gauda EB, McLemore GL, Tolosa J, Marston-Nelson J, Kwak D. Maturation of peripheral arterial chemoreceptors in relation to neonatal apnoea. Semin Neonatol. (2004) 9:181-94. doi: 10.1016/j.siny.2003.11.002

11. van Leuteren RW, Kho E, de Waal CG, Te Pas AB, Salverda HH, de Jongh FHC, et al. Cardiorespiratory monitoring in the delivery room using transcutaneous electromyography. Arch Dis Child Fetal Neonatal Ed. (2020). doi: 10.1136/archdischild-2020-319535. [Epub ahead of print].

12. Dawson JA, Kamlin COF, Vento M, Wong C, Cole TJ, Donath SM, et al. Defining the reference range for oxygen saturation for infants after birth. Pediatrics. (2010) 125:1510. doi: 10.1542/peds.2009-1510

13. Wyckoff $\mathrm{MH}$, Aziz K, Escobedo MB, Kapadia VS, Kattwinkel J, Perlman JM, et al. Part 13: neonatal resuscitation: 2015 American Heart Association guidelines update for cardiopulmonary resuscitation and emergency cardiovascular care. Circulation. (2015) 132:S543-60. doi: 10.1161/CIR.0000000000000267

14. Prechtl HFR, Van Eykern LA, O’Brien MJ. Respiratory muscle EMG in newborns: a non-intrusive method. Early Hum Dev. (1977) 1:265-83. doi: 10.1016/0378-3782(77)90040-8

15. Lopes J, Muller NL, Bryan MH, Bryan AC. Importance of inspiratory muscle tone in maintenance of FRC in the newborn. J Appl Physiol. (1981) 51:830-4. doi: 10.1152/jappl.1981.51.4.830

16. Pellegrini M, Hedenstierna G, Roneus A, Segelsjö M, Larsson A, Perchiazzi G. The diaphragm acts as a brake during expiration to prevent lung collapse. Am J Respir Crit Care Med. (2017) 195:1608-16. doi: 10.1164/rccm.201605-0992OC 
17. Pas ABTE, Siew M, Wallace MJ, Kitchen MJ, Fouras A, Lewis RA, et al. Establishing functional residual capacity at birth: the effect of sustained inflation and positive end-expiratory pressure in a preterm rabbit model. Pediatr Res. (2009) 66:295-300. doi: 10.1203/PDR.0b013e3181b1bca4

18. Kraaijenga JV, Hutten GJ, De Jongh FH, Van Kaam AH. The effect of caffeine on diaphragmatic activity and tidal volume in preterm infants. J Pediatr. (2015) 167:70-5. doi: 10.1016/j.jpeds.2015. 04.040

19. te Pas AB, Hooper S. Initiation of breathing at birth. In: Jobe AH, Whitsett JA, Abman SH, editors. Fetal and Neonatal Lung Development: Clinical Correlates and Technologies for the Future. Cambridge: Cambridge University Press (2016). p. 164-86.

20. Caravagna C, Seaborn T. Oxygen sensing in early life. Lung. (2016) 194:71522. doi: 10.1007/s00408-016-9908-x

21. Dunwiddie TV, Masino SA. The role and regulation of adenosine in the central nervous system. Annu Rev Pharmacol Toxicol. (2001) 41:145-74. doi: 10.1146/annurev.neuro.24.1.31

22. Hepple RT. The role of $\mathrm{O}_{2}$ supply in muscle fatigue. Can J Appl Physiol. (2002) 27:56-69. doi: 10.1139/h02-004

23. Te Pas AB, Wong C, Kamlin COF, Dawson JA, Morley CJ, Davis PG. Breathing patterns in preterm and term infants immediately after birth. Pediatr Res. (2009) 65:352-6. doi: 10.1203/PDR.0b013e31819 $3 \mathrm{f} 117$
24. Keens TG, Bryan AC, Levison H, Ianuzzo CD. Developmental pattern of muscle fiber types in human ventilatory muscles. J Appl Physiol Respir Environ Exerc Physiol. (1978) 44:909-13. doi: 10.1152/jappl.1978.44.6.909

25. Oda A, Parikka V, Lehtonen L, Soukka H. Rapid respiratory transition at birth as evaluated by electrical activity of the diaphragm in very preterm infants supported by nasal CPAP. Respir Physiol Neurobiol. (2018) 258:1-4. doi: 10.1016/j.resp.2018.09.009

26. Oei JL, Finer NN, Saugstad OD, Wright IM, Rabi Y, Tarnow-Mordi W, et al. Outcomes of oxygen saturation targeting during delivery room stabilisation of preterm infants. Arch Dis Child Fetal Neonatal Ed. (2018) 103:446-54. doi: 10.1136/archdischild-2016-312366

Conflict of Interest: The authors declare that the research was conducted in the absence of any commercial or financial relationships that could be construed as a potential conflict of interest.

Copyright $\odot 2021$ van Leuteren, Scholten, Dekker, Martherus, de Jongh, van Kaam, te Pas and Hutten. This is an open-access article distributed under the terms of the Creative Commons Attribution License (CC BY). The use, distribution or reproduction in other forums is permitted, provided the original author(s) and the copyright owner(s) are credited and that the original publication in this journal is cited, in accordance with accepted academic practice. No use, distribution or reproduction is permitted which does not comply with these terms. 